\title{
Spiral Arms in an Infant Galaxy
}

\author{
Astronomers have identified spiral arm features in a galaxy from the early \\ Universe, suggesting that these arms can develop more rapidly than some \\ theories predict.
}

By Michael Schirber

W hen imagining a galaxy, we often picture it with lovely spiral arms. But how these spiral features form remains unclear. Are spiral arms the final step in some long, steady development, or do they come and go in cycles, like "galactic seasons"? One way to answer these questions is to study the earliest spiral galaxies in the Universe. A new study, appearing in Science, has identified the earliest-yet-observed spiral arm features in a galaxy. The timing of the arms' appearance-1.4 billion years after the big bang-suggests that spiral galaxies can develop more rapidly than some galactic models predict.

Spiral galaxies are common in the nearby Universe, with roughly $70 \%$ of observed galaxies, including our Milky Way, sporting spiral arms. But as astronomers look farther away-or, equivalently, back in time-the percentage of spirals appears to

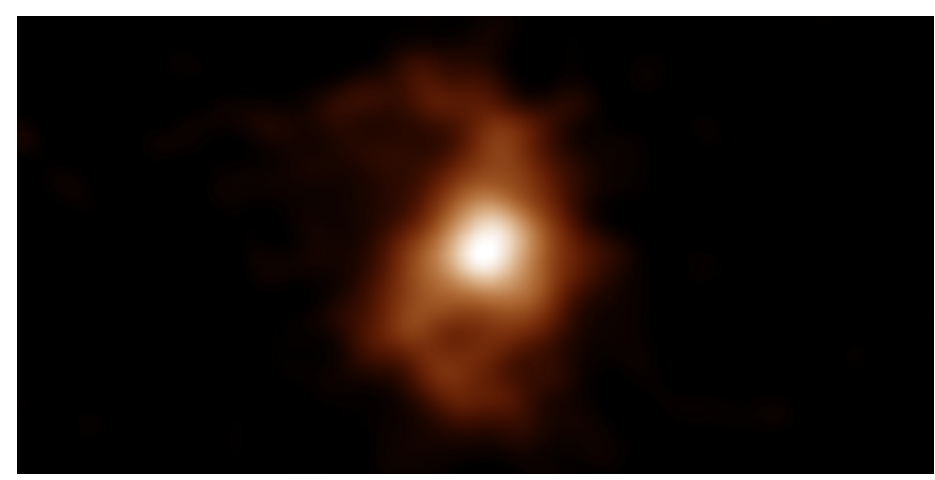

This image from the ALMA observatory shows the emission from carbon ions in the galaxy BRI 1335-0417. The emission map reveals spiral arm features that are the earliest observed, appearing just 1.4 billion years after the big bang.

Credit: ALMA (ESO/NAOJ/NRAO); T. Tsukui and S. Iguchi decrease. "It may be due to the observational limitation-we need high angular resolution and sensitivity to identify spiral structures in observed images," says coauthor Takafumi Tsukui, a graduate student from SOKENDAI, the Graduate University for Advanced Studies in Japan.

But it might be that spiral structures can't form in young galaxies, as suggested by the currently prevailing theory for spiral arms, spiral density wave theory. This theory suggests that arms can only emerge in galaxies that had time to settle down into stable, rotating disks. "Theorists believe it takes a long time to form spiral arms," says coauthor Satoru Iguchi, an astronomer from the National Astronomical Observatory of Japan. Exactly how long is still a matter of debate.

Previous work had spotted spiral arm features in a galaxy from 11.3 billion years ago. Since the galaxy must have formed sometime after the big bang (13.8 billion years ago), spiral arm formation would have to be faster than about 2 billion years. Tsukui and Iguchi cut this formation time window by roughly half by identifying spiral arm morphology in an even earlier galaxy. The astronomers were studying archive data from the Atacama Large Millimeter/submillimeter Array (ALMA) - a radio observatory in Chile. In particular, they were looking at a handful of galaxies whose light emission includes lines from ionized carbon, which resides in gas clouds around young stars. One of the galaxies, BRI 1335-0417, appeared to have two spiral arms stretching out about 15,000 light years from the center.

The spiral features appear prominent at carbon wavelengths because the ion emission is concentrated in regions of dense stellar activity, such as spiral arms. To confirm the presence of the arms, Tsukui and Iguchi further analyzed data from this galaxy, obtaining the velocity of material in different regions of 
the galaxy. This velocity map showed that BRI 1335-0417 has a rotating disk, as one would expect for a spiral galaxy. The map also revealed that the velocity is uniform along the arms, evidence that they are bound structures within the galaxy.

Galaxy expert Andreas Burkert from Ludwig Maximilian University of Munich says that it is interesting that a spiral galaxy appears so early in the Universe, when galaxies are expected to have been frequently merging with other galaxies. He explains that spiral arms are believed to form out of gravitational instabilities within a galaxy disk. But these instabilities can only grow into arms when the disk has reached a kind of equilibrium between the forces of gravity, pressure, and shear. "There is some fine-tuning needed," Burkert says, which the observed disk appears to have achieved, according to the analysis by Tsukui and Iguchi. "However, it is surprising that this young disk has self-regulated to the disk equilibrium state," Burkert says. Whether this early regulation is rare or not, he can't say, as simulations of the early Universe are not yet detailed enough to compute the dynamics within galactic disks.

But perhaps equilibrium conditions aren't necessary, says Jerry Sellwood from the University of Arizona. Sellwood has worked on spiral arm models for 40 years, and today he believes that spiral arms can form under less quiescent conditions. "The galaxy formation process is messy," he explains. And that messiness leads to perturbations that can induce spiral patterns that come and go with a rapid turnover rate. "The Milky Way probably has had something like 25 different spiral patterns in its lifetime," Sellwood says. This recurrent spiral model could easily explain a galaxy having spiral arms within a billion years of its formation.

However, Sellwood says that another possibility is that BRI 1335-0417's spiral arms formed from a galactic encounter. "It looks to me very much like a companion whizzed by the observed galaxy," he says. Such a tidal interaction could explain the unusually strong spiral arms in the ALMA observations.
Sellwood thinks BRI 1335-0417 could be an early-Universe analog of the stunningly beautiful Whirlpool Galaxy, a nearby spiral galaxy that is clearly being "stirred" by a small companion galaxy.

Tsukui and Iguchi considered a tidal origin for BRI 1335-0417, but they think this scenario is disfavored because it normally

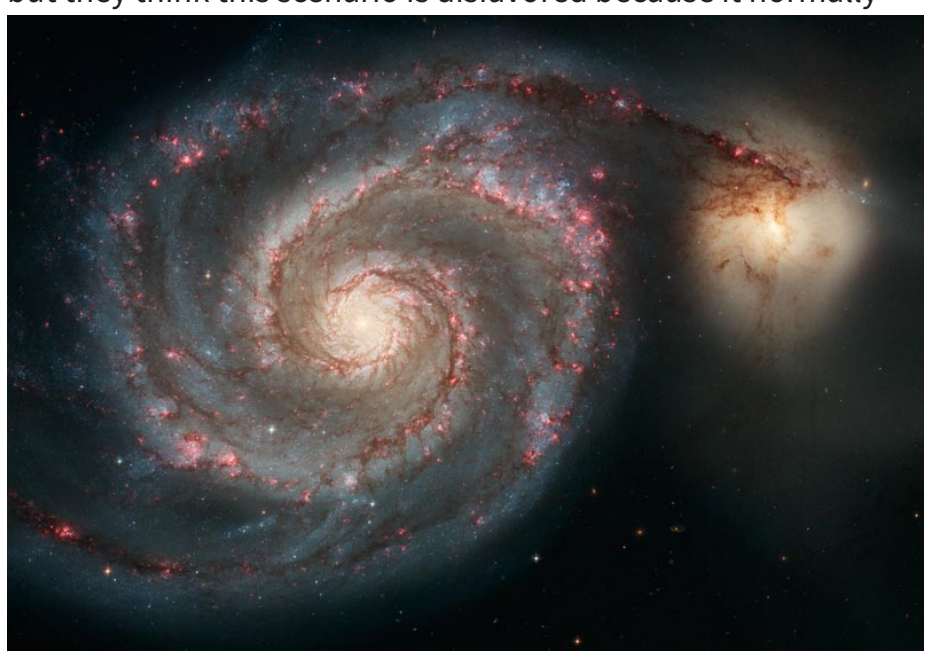

The Whirlpool Galaxy, or M51, lies about 100 million light years from us. The small companion galaxy seen to the right is thought to have induced the spiral structure in the larger galaxy through tidal interactions.

Credit: S. Beckwith (STScl) Hubble Heritage Team, (STScl/AURA), ESA, NASA

produces long tidal tails, which are not observed. Higher resolution images of BRI 1335-0417 could help uncover the circumstances behind this early galaxy's show of arms.

Michael Schirber is a Corresponding Editor for Physics based in Lyon, France. 